\title{
NEW TRENDS IN PERSONALITY PSYCHOLOGY: SOCIAL AND VIRTUAL ASPECT
}

\author{
Tatiana Martsinkovskaya ${ }^{1}$, Daria Tkachenko ${ }^{2}$, \& Vladimir Karpuk ${ }^{2}$ \\ ${ }^{1}$ Institute of Psychology, Russian State University for the Humanities, 125993, Miusskaya square 6, \\ Moscow, Russia; Psychological Institute RAE, 125009 Mochovaya 9, Moscow (Russia) \\ ${ }^{2}$ Russian State University for the Humanities, 125993, Miusskaya square 6, Moscow (Russia)
}

\begin{abstract}
Psychology is currently facing global challenges that with necessity lead to the emergence of fundamentally new trends and patterns in the theory and practice of personality psychology. From the point of view of theory, there is a constant rethinking of changes in the structure and content of identity - personal, sociocultural, ethnic. In practice, there are no less significant processes associated with approaches and methods in diagnostics and counseling. These changes are associated with the expansion of the virtual space of identification and self-realization. In the last year, the changes associated with quarantine for COVID 19 have become of great importance. The frustration of real space, which often connects with a narrowing of the time perspective, leads not only to an increase in the role of virtual space, but also to intensification of the role of network identity and the development of various forms of Internet communication, counseling and leisure activities. It appears that new trends will become more significant and constant in the future. Therefore, it is imperative to discuss the new forms of narrative and virtual identity, the directions for further change and their positive and negative impact on the identification and well-being of both young and old people.
\end{abstract}

Keywords: Frustration of space and time, wellbeing, identity, narrative.

\section{Introduction}

During the last year life has put on an impressive natural experiment in which we have met a variety of challenges: a new incomprehensible somatic illness, physical and mental burnout, a difficult economic and environmental situation, fatigue from the uncertainty of the situation and the rigidity of barriers that have radically changed the space and time of our life. ... And all these challenges require a quick response, because the delay in the reaction can turn into a fan of new problems.

The shock, the suddenness of change and the emergence of a fundamentally new situation, of course, raises the motivation to preserve our-selves, our values and lifestyle. As the search activity of people is intensified, it is very important to analyze the preferred information resources and the degree of trust in them. Indeed, it is the degree of trust that largely determines the degree of influence of this information on behavior. Another problem is the relationship between psychological and economic precariousness. (Druzhilov, 2015).

Analyzing the psychological impact of quarantine scientists mention as difficulties in both coming in and out of the quarantine situation and the influence of uncertainness. (Brooks \& others, 2020). (Jacobson \& others, 2020). In a situation of frustration new forms of communication appear the importance of social networks, telephone and Internet communication platforms increases. In a quarantine situation, not only conferences, lectures and exams moved to the Internet, but also friendly conversations and even meals, including the celebration of significant events. Although, of course, such communication cannot replace the real one, nevertheless, it is an important substitute for it, helping to maintain emotional contact, psychological well-being and the necessary sources of information. (Martsinkovskaya, 2019, 2019a). In this context, a person's ability to accept changes and tolerance for uncertainty become extremely important (Grishina, 2019). Also, there must be taken into account the latest publications on the coping problem, especially, issues related to the difference in individual reactions in retroactive and proactive coping (Frydenberg, 2014).

At the same time, one can assume a gradual adaptation to the new reality and even the discovery of positive aspects in it. Therefore, it is necessary to constantly monitor the situation and fix all emotional, personal and behavioral changes.

\section{Design, method, participants.}

The aim of this work was to study mental well-being, behavior and emotional experience of a pandemic and quarantine in a situation where real interactions were replaced by a virtual one. 
The study involved students of Moscow universities $(17-23, \mathrm{n}=90)$ and people of mature age $(45-60, n=60)$. The survey was conducted with the help of social networks; all respondents were aware of its purpose and agreed to participate in the work.

The questions were divided into several groups, for each of which the received answers were processed separately.

1. Attitude to information

This group included questions aimed at studying the main information sources that respondents used to find out news about the situation about the pandemic, the specifics of the virus, the characteristics of the course of the disease and methods of prevention.

2. Emotional state

This group included questions that reveal the attitude of people to the quarantine situation, changes in the nature of communication, study, work and entertainment. When analyzing the answers, possible ways of replacing emotional contacts and options for spending leisure time were also recorded.

3. Behavior

When analyzing the answers to these questions, both methods of behavior in everyday life (food, masks, gloves) and the relationship between real and virtual behavior, especially during study / work and entertainment, were analyzed.

4. Visions of the future

This group of questions was aimed on analyzing the respondents' ideas about their future after the end of quarantine, as well as about the role of new forms of communication for the future of the whole world.

\section{Discussion}

Contrary to expectations, no significant differences were found in the sources of information between the respondents of both age groups. Almost everyone searched for information in the Internet, and trusted this information more than TV. Apparently, when it comes to information that is important for life, not only young people, but also adults and the elderly are trying to get data from various sources and various Internet resources. The differences were mainly due to the fact that adults mostly identified social networks, instant messengers and the Internet in general, while students focused on specific messengers, social networks and, especially, streaming video. At the same time, young people trust their friends and adults are more focused on the opinions of famous people.

No significant difference was found in $n$ the behavior of people in both age groups. All of them agreed with necessity to maintain social distance and wear masks, although this prescription is not always fully implemented. The only things that really take place are hand washing and the use of disinfectants and wet wipes. At the same time, both groups are irritated by the need to comply with these strict regulations. These restrictions are especially annoying to adults and the elderly. They also often wear groceries and try to minimize contacts.

The main difference in the emotional state of the young and the elderly is related to the time perspective. Changes in the daily routine of life are already affected work and communication with family and friends, and even leisure activities. Elderly people experience depression not so much because of the quarantine situation and movement restrictions, but because of the fear that this situation may become permanent for them for the rest of their lives. This situation reduces the time perspective and darkens the idea of the future.

Young people are less prone to depression and do not consider quarantine as the factor, that can influence the time perspective and change their global plans for the future. The preservation of emotional stability among young respondents is also facilitated by the fact that they were able to find a substitute for real communication and spending their leisure time in virtual space. Older respondents communicate with family and friends mostly by phone, less often via Skype, and even less often by Zoom. Some watch old films or performances, a few - concerts or virtual tours of museums. Young people have much wider opportunities provided by the Internet space. These are online training courses, which many actively use in addition to the basic courses at the university, as well as the emergence of new hobbies and new ways of spending leisure time, when they play together and even dance (zoom parties, discussion of their streaming videos, etc.).

Another important point is related to the assessment of new technologies for the future world. Here we can state a difference in the predominantly positive attitude of young people, who answered that they hope for changes for the better and noted the importance of new technologies in modernizing the world. For adults and the elderly, expectations are more reserved and the prospects for change are assessed as ambivalent. For many adults, changes are associated with psychological and economic precariousness. In general, the first data is presented on figure 1. 
Figure 1. The ratio of real and Internet space.

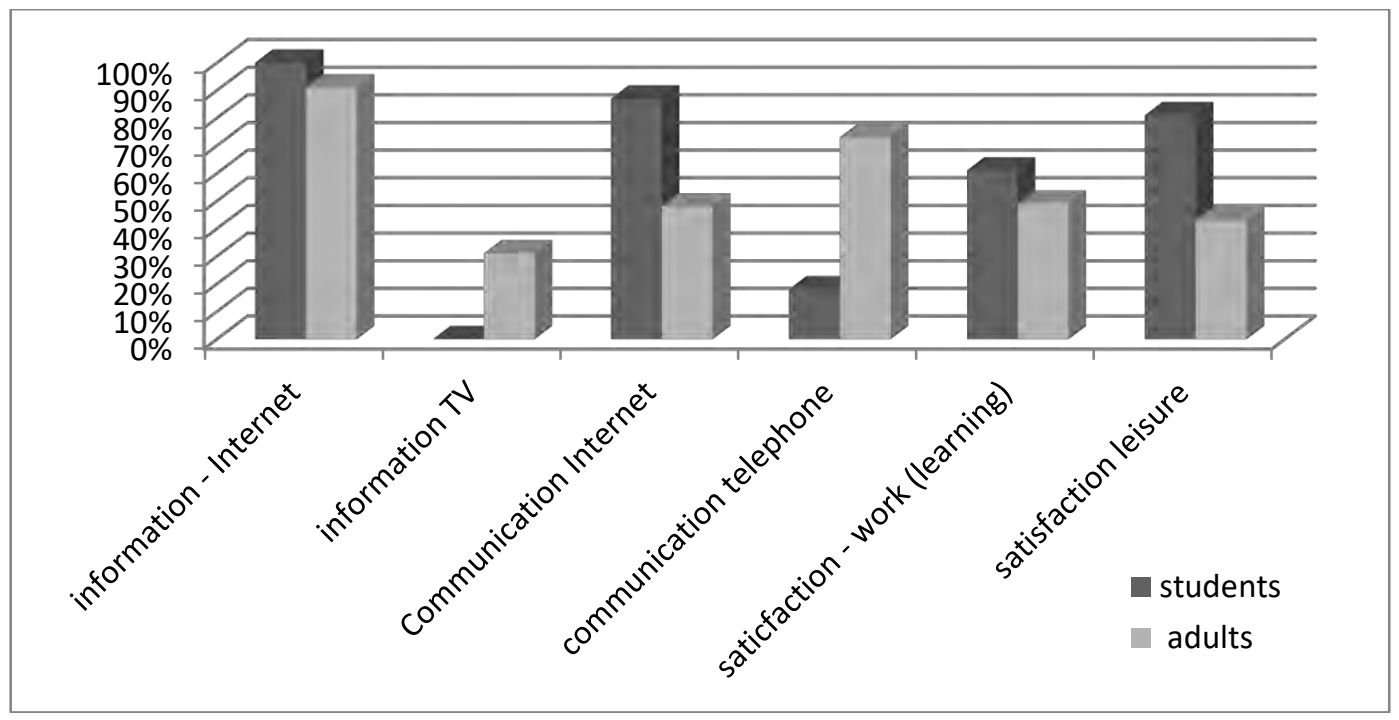

Of course, the psychological state of many people, regardless of age, is not cloudless, even people with a predominantly positive attitude have moments of irritation, fatigue, depression. However, even a small monitoring shows a slight increase in positive well-being, primarily, an increase in the positive assessment of online work and learning.

\section{Conclusions}

The obtained data showed that in a difficult situation trust in information coming from the Internet is significantly higher than to information from others sources. Apparently, the importance of the situation leads to the fact that the ability to choose an interesting and significant channel increases the role of the Internet for all groups of the population.

The difference in the emotional state is only partially and indirectly determined by age, since young people are more flexible in using the virtual space to replace real options for communication, study and entertainment.

The materials obtained confirm that at present, the attitude towards the information and cultural space of the Internet significantly affects the boundaries of the real social field, allowing one to overcome various types of external frustration, and often even the most severe barriers.

\section{Acknowledgments}

This work was supported by the Russian Science Foundation, project 19-18-00516 "Transitive and virtual spaces - commonality and differences".

\section{References}

Brooks, S. K. \& others (2020). The psychological impact of quarantine and how to reduce it: rapid review of the evidence. Lancet, 14(395), 912-920, https://doi.org/10.1016/S0140-6736(20)30460-8

Druzhilov, S.A. (2015). Precariat and informal employment in Russia: social and psychological aspects. Humanitarian research, 41(1-2), 53-61

Frydenberg, E. (2014). Coping research: Historical background, links with emotion, and new research directions on adaptive processes. Australian Journal of Psychology, 66, 82-92.

Grishina, N.V. (2019). Procedural approach in personality psychology. Psychology of personality: Stay in change. SPb.: SPbSU publishing house

Jacobson, N.C. Lekkas, D. \& others (2020) Mental health search: COVID-19stay-at-home orders. JMIR Mental Health, 7(6), e1934. doi:10.2196/19347

Martsinkovskaya, T. D. (2019). Person in Transitive and Virtual Space: New Challenges of Modality. Psychology in Russia: State of the Art, 12(2), 165-176. DOI: 10.11621/pir.2019.0212

Martsinkovskaya, T. D. (2019a). Information space of a transitive society: problems and development prospects. Consultative psychology and psychotherapy. 27 (3), 77-96, doi: 10.17759 / cpp.2019270306 